\title{
PLANTS
}

\section{A PRELIMINARY FLORAL LIST FOR THE RENDEK ELM FOREST NATURE SANCTUARY IN EAST-CENTRAL SASKATCHEWAN}

VERN HARMS, 212-115 Keevil Crescent, Saskatoon, SK S7N 4L9 (Phone: 306-373-0097) and LES BAKER, Box 90, Carragana, SK SOE OKO (Phone: 306-278-2172)

One of Nature Saskatchewan's six nature preserves is the Rendek Elm Forest located about $121 / 2 \mathrm{~km}$ northeast of Erwood (or $25 \mathrm{~km}$ east-northeast of Hudson Bay) at the confluence of Smoking Tent Creek with the Red Deer River. It is found at the latitude-longitude coordinants of $52^{\circ} 54^{\prime} \mathrm{N}, 102^{\circ} 01^{\prime}-02^{\prime} \mathrm{W}$, and the survey coordinants of SW $1 / 4$ Sec. 25 and E edge of SE Sec. 26, Twp. 45 , Rge. $01, W 2^{\text {nd }} M$.

We have noted Nature Saskatchewan's expressed interest in compiling bioinventories of their various nature sanctuaries (Nature Views, \#114, SpringSummer 1998, p. 13). This is a worthwhile objective with which we whole-heartedly concur. Also noted was their request for volunteers to help document the plants and animals at any of these sites. As a contribution towards this goal, we wish to report on a plant collection made on 9 July 1981 at one of these nature preserves the "Rendek Elm Forest" - by the present authors along with Donald Hooper and Patricia Sky.

Recorded from this site at that time were 52 species of vascular plants, belonging to 49 genera, in 30 families. Two species were pteridophytes (i.e. free-sporing plants), with the rest being angiosperms (i.e. flowering plants). The following four recorded species have been assigned a provincial rarity status: Blue Monkey-flower (Mimulus ringens), Nodding Trillium (Trillium cernuum), Assiniboia Sedge (Carex assiniboinensis), and Red Elderberry (Sambucus racemosa ssp. pubens). All records were documented by voucher specimens mounted and filed in the Fraser Herbarium (SASK) at the University of Saskatchewan.

The species found are listed below in alphabetical order of their scientific names under their respective families, which in turn are alphabetically arranged. The species' scientific names are followed by their authors, pertinent synonyms (if any), common name(s), and a brief, single-word, general habitat descriptor. The primary general habitat (indicated as "woods") is an extensive, moist, flood-plain woods with the canopy dominated by American EIm and Manitoba Maple, and with a lush herb layer largely dominated by dense Ostrich Ferns. A second general habitat type (indicated as "bank") is the more limited extent of open to semi-open, gravelly river-bank. A third general habitat type (indicated as "shoreline") is the marsh-edged, river shoreline. Of the 52 species recorded by us at the Rendek Elm Forest site, 32 were from 
the woods", 15 from the "bank," and 5 from the "shoreline" habitat types present there. For the four rare plants, the provincial rarity status assigned to them by the Saskatchewan Conservation Data Centre is added in parentheses.

With our botanizing at the site for only a few hours of one day, this represents a quite preliminary survey. A more intensive floristic inventory, continued over the entire growing season, would no doubt increase the species' list to perhaps double, if not even to three or four times the number listed here.

Our July 1981 visit and preliminary botanical survey of the Rendek Elm Forest site probably had a historical significance beyond the plants recorded, since it, along with other visits made by him, inspired one of our participants, Donald Hooper, to propose the area to the Hudson Bay Regional Park and Nature Saskatchewan for protection as a nature sanctuary.

\section{SPECIES LIST}

\section{Aceraceae (Maple Family):}

Acer negundo L.; MANITOBA MAPLE, BOX-ELDER. Woods. (A codominant tree.)

Alismaceae (Water-plantain Family): Alisma plantago-aquatica L. var. americana Schultes and Shultes; BROADLEAF WATER-PLANTAIN. Shoreline.

Sagittaria latifolia Willd.; BROADLEAVED ARROWHEAD. Shoreline.

Apiaceae (Umbelliferae) (Carrot Family):

Heracleum lanatum Michx.; COW PARSNIP. Bank.

Osmorhiza longistylis (Torr.) DC.; SMOOTH SWEET CICELY. Woods.

Apocynaceae (Dogbane Family):

Apocynum cannabinum L. var. hypericifolium Gray [A. sibiricum Jacq.]; INDIAN HEMP; CLASPING-LEAF DOGBANE. Bank.
Aspleniaceae (Spleenwort Family):

Matteuccia struthiopteris (L.) Todaro;

OSTRICH FERN. Woods. (A predominant understory forb.)

Asteraceae (Compositae) (Aster Family):

Antennaria parvifolia Nutt.; LOW or SMALL-LEAF PUSSYTOES. Bank.

Erigeron philadelphicus L.; PHILADELPHIA FLEABANE. Woods. Eupatorium maculatum L.; SPOTTED JOE-PYE WEED. Bank.

Helenium autumnale L. var. montanum (Nutt.) Fern.; SNEEZEWEED. Woods. Rudbeckia hirta L. var. pulcherrima Farw. [R. serotina Nutt.]; BLACK-EYED SUSAN. Bank.

Brassicaceae (Cruciferae) (Mustard Family):

Erysimum cheiranthoides L.; WORMSEED MUSTARD. Woods.

Cannabinaceae (Hemp Family):

Humulus lupulus L.; COMMON HOP. Woods.

Caprifoliaceae (Honeysuckle Family): Sambucus racemosa L. ssp. pubens (Michx.) Hulten; RED ELDERBERRY. Woods. (S3).

Symphoricarpos albus (L.) Blake; NORTHERN SNOWBERRY. Woods.

Viburnum edule (Michx.) Raf.; LOW BUSH-CRANBERRY. Woods.

Viburnum opulus L. ssp. trilobum (Marsh) Clausen; HIGH BUSHCRANBERRY. Woods.

\section{Caryophyllaceae (Pink Family):}

Cerastium nutans Raf.; LONGSTALKED CHICKWEED. Bank.

Stellaria longifolia Muhl.; LONGLEAVED CHICKWEED, STARWORT or STITCHWORT. Woods.

Convolvulaceae (Morning-glory Family):

Convolvulus sepium L.; HEDGE BINDWEED; WILD MORNING-GLORY. Bank.

Cyperaceae (Sedge Family):

Carex assiniboinensis Boott; ASSINIBOIA SEDGE. Woods. (S2). Carex retrorsa Schwein.; TURNED or 
RETRORSE SEDGE. Woods.

Scirpus validus Vahl.; GREAT BULRUSH. Shoreline.

Equisetaceae (Horsetail Family):

Equisetum arvense L.; COMMON or FIELD HORSETAIL. Woods.

Grossulariaceae (Currant Family):

Ribes americanum Mill.; WILD BLACK CURRANT. Woods.

Hippuridaceae (Mare's-tail Family):

Hippuris vulgaris L.; MARE'S-TAIL. Shoreline.

Juncaceae (Rush Family):

Juncus nodosus L.; KNOTTED RUSH. Bank.

Lamiaceae (Labiatae) (Mint Family): Lycopus americanus Muhl.; CUT-LEAF WATER-HOREHOUND. Woods.

Mentha arvensis L.; WILD MINT. Woods.

Stachys palustris L. ssp. pilosa (Nutt.) Epling.; MARSH HEDGE-NETTLE. Bank. Liliaceae (Lily Family):

Smilacina stellata (L.) Desf.; STARFLOWERED SOLOMON'S-SEAL. Woods.

Trillium cernuum L.; NODDING TRILLIUM or WAKE-ROBIN. Woods. (S2/S3).

\section{Onagraceae (Evening-primrose} Family):

Circaea alpina L.; ENCHANTER'S NIGHT-SHADE. Woods.

Oenothera biennis L.; YELLOW EVENING-PRIMROSE. Bank.

Poaceae (Gramineae) (Grass Family): Glyceria grandis Wats.; TALL MANNAGRASS. Shoreline.

Poa palustris L.; FOWL BLUE-GRASS. Woods.

Polygonaceae (Buckwheat Family): Rumex triangulivalvis (Dans.) Rech. f. [R. mexicanus Meisn.; R. salicifolius Weinm.]; NARROW-LEAVED or WILLOW DOCK. Bank.

Primulaceae (Primrose Family):

Lysimachia ciliata L.; FRINGED LOOSESTRIFE. Woods.

Ranunculaceae (Buttercup Family):

Actaea rubra (Ait.) Willd.; BANEBERRY.
Woods.

Anemone canadensis L.; CANADA ANEMONE (or WIND-FLOWER). Bank. Ranunculus abortivus L.; SMALLFLOWERED CROWFOOT or BUTTERCUP. Woods.

Ranunculus macouniiBritt.; MACOUN'S BUTTERCUP. Woods.

Thalictrum dasycarpum Fisch. \& Lall.; TALL MEADOW-RUE. Bank.

Rosaceae (Rose Family):

Prunus virginiana L.; CHOKE-CHERRY.

Woods.

Rubiaceae (Madder Family):

Galium triflorum Michx.; SWEETSCENTED BEDSTRAW. Woods.

Salicaceae (Willow Family):

Salix exigua Nutt. [S. interior Rowlee]; SANDBAR WILLOW. Bank.

Scrophulariaceae (Figwort Family):

Mimulus ringens L.; BLUE MONKEYFLOWER. Bank. (S1).

Veronica americana (Raf.) Schw.; AMERICAN SPEEDWELL. Woods.

Ulmaceae (EIm Family):

UImus americana L.; AMERICAN ELM. Woods. (A dominant or codominant tree.) Urticaceae (Nettle Family):

Urtica dioica L. ssp. gracilis (Ait.) Seland. [U. gracilis Ait.; U. procera Muhl.; U. Iyallii S. Wats.]; STINGING NETTLE. Woods.

Violaceae (Violet Family):

Viola rugulosa Greene. [V. canadensis] WESTERN CANADA VIOLET. Woods.

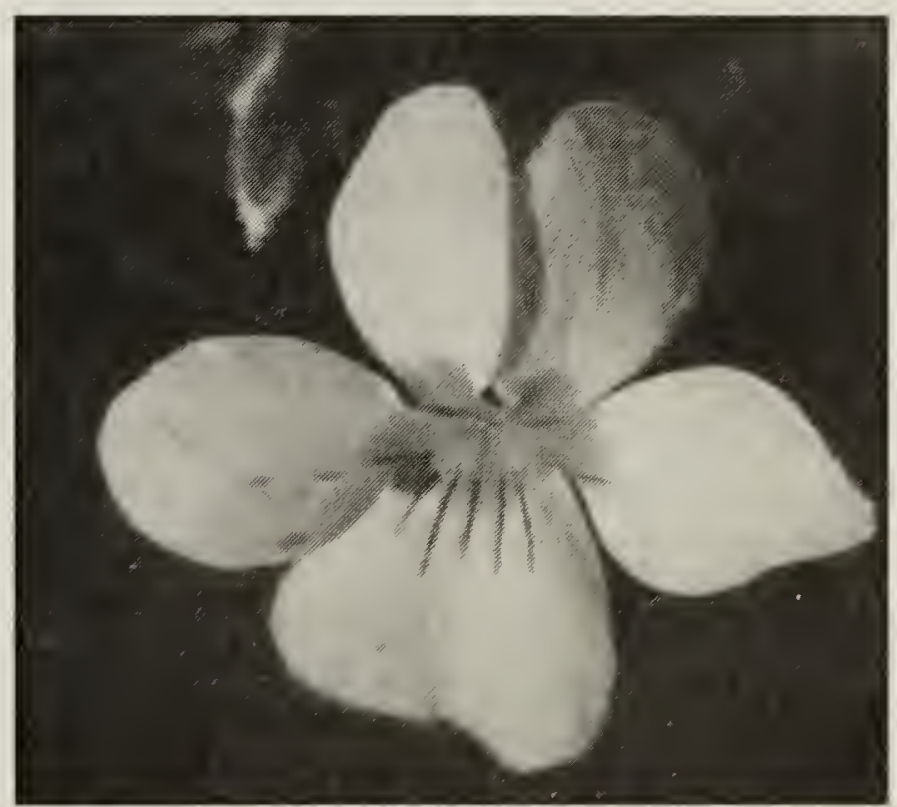

Western Canada Violet

G.H.Hardy 